\title{
Footwork Teaching of College Badminton Elective Course Chao CHEN
}

\author{
Liuzhou Railway Vocational Technical College, Liuzhou, 545007, China \\ Email: ccyd1970@163.com
}

Keywords: badminton teaching, footwork, technique

\begin{abstract}
Badminton Footwork is an important part of the badminton technology, learning and mastering the quick and accurate method is to lay the important link of badminton. In the process of actual learning badminton technology, people often focus on technique study but do not care too much footwork training, it can not be guaranteed to study results. Therefore, it is necessary to strengthen the footwork training in the college badminton teaching, this paper puts forward several effective teaching methods in the course of Badminton Footwork training.
\end{abstract}

\section{Introduction}

Badminton is one of favorite elective course of young college students .characteristics of badminton movement is that step is the basis,however, in the usual teaching,I found that the students lack of understanding of the importance of footwork , and they just focus on learning action on the hand, not too much attention to footwork learning, if the teachers accommodate themselves to students in teaching, Not enough to footwork learning requirements and the degree of attention, focus on technique study but do not care too much footwork training. this result is clearly not to increase the level of skills and tactics of badminton. In fact, only the well-placed footwork can ensure the integrity of the technique, in order to improve the teaching quality of badminton, we must pay attention to badminton footwork teaching.

\section{Basic Knowledge of Badminton Footwork}

Footwork is the soul of badminton. Badminton footwork to move around the direction can be divided into three categories: one is the volleying step, two is the backward step ,three is the midfield transverse moving step . Every complete footwork, including starting, moving, Assist in the completion of the ball and reverse. Starting is a process that turn the relatively static stance state to force the direction of ball moves, it comes from the judgment and reaction. Moving is generally only by the center position to the position of hitting the ball . Brake is to restraint the inertia of moving when you reached the position, and keep the body center of the stable, in order to assist the bating action. The reverse is back to the centre as soon as possible after the completion of the stroke,get ready to meet the next ball. These footwork what used in above like crossing step, thrust step, stride and jumping form the method of moving in the court .Students are required to take notice of the cohesion of judgment, starting, mobile, reversing in practice.

\section{The Method of Footwork Teaching}

\section{Teaching of Manual Footwork Decomposition Training}

Badminton Footwork includes the volleying step, the backward step and the midfield transverse moving step. In the early step teaching, we can separately to explain the front of volleying, midfield catching and backfield backward moving method by manual footwork decomposition, let the students back to the front line and repeated footwork, Practice both sides moving, the four angle footwork to low center of gravity forward and backward. experience action carefully. On this basis, combine the manual decomposition footwork exercises and actual combat experience of hitting ,coordination 
center of gravity, moving time and hand batting action. As an example,up close to the net, starting from the center position to hit the ball right up front, one toss the ball for practitioners,practitioners consciously forward the net and moving the left foot-- cross step forward the ball movement -"patting" -- back toward the center position by the right foot trusting . Repeated moving in batting practice, familiar with the consolidation of footwork. Teachers should take the skill of stride crossing step and thrust step are repeatedly practicing bare handed. With the depth of teaching, combining with practice the multi ball technique to strengthen skill, to .enable students to know select the step according to the ball moving direction, at the same time, continuously improve the proficiency degree and movement speed.

\section{The Connection Method of Teaching}

Connecting ball footwork refers to the use of at least two different footwork in the batting process, the key of teaching is the organic combination of different footwork, to improve footwork coherence. We should follow the principle that from easy to difficult in footwork teaching, and use the mixed foot works to practice Repeatedly.For example, frontcourt footwork connect midfield play,backcourt footwork connect up to the net,frontcourt footwork connect the backcourt batting, backcourt footwork connect midfield play backhand footwork connect the midfield smash.Combined the front,midfield and backfield footwork with the actual combat and practice to constantly improve footwork coherence and flexibility.

\section{The Combination of Method and Footwork}

The rational use of methods is the footwork is the basic premise of, only through the footwork to seize a favorable position, to ensure the batting action of integrity, if you have good footwork but do not have the good performance, you also could not play well, therefore, the badminton technique and footwork is the two necessary conditions to learn badminton, they mutual dependence and mutual connection, they are indispensable, as mentioned before, The basic elements of step contained on any batting action, in coordination with the strength, speed and distance, step and means are inseparable to form a whole, especially in combination with body weight support, conversion, balance, technique and step reflects more prominent, combining with manual footwork teaching throughout the whole teaching. Thus, we should pay attention to the students when they hit the ball in classes, do not confined to look for reasons from the methods, and take into account the influence of footwork caused by stroke technique action. Such as step is not in place, before kicking, pushing ball and cartwheel block in front of the net are very easy to mistake, they even unable to complete the actions. When learning the technology of front net, linking up the front net footwork up and using practice of multi ball, improve methods and footwork synchronously. Similarly, when you learn the lofty ball, link up the lofty ball and backcourt footwork, students practice with the combination of methods and footwork in class won't be bored, this teaching idea is more aggressive, and makes a better teaching effect.

\section{Combining Line and Coordinating Tactical with Footwork in Teaching}

Footwork learning has reached a certain stage, combined with the line and the tactics, some special coherent footwork practice has practical value, footwork is a relatively smooth, faster, it will help you to grab the good shotting position, then you batting will be more threat. For examples, the smashing in front of the net and repeat ball, some fixed line and using running practice. Through these combinations of lines and footwork to practice and improve the ability and use consolidate footwork continuously.

\section{Clear Footwork and Pay Attention to the Accuracy and Completeness of Actions}

Attention should be paid to ensure that students learning step when the basic step action of normative and integrity, it is to let the students can take other actions, including the methods, footworks, batting ball and fake to lay the foundation for comprehensive application. The students common problems in step learning including not timely return after striding and hitting, using bad angle in the moving process, curved running and etc... only to master correct gist of step, then the students can improve footwork levels through effective practice, that requires teachers order 
corrective training in the class to make students aware that is the most important to maintain their center of gravity in badminton footwork moving, so the center of gravity is relatively stable, and make the fluctuation range of the process of running suitable, it can reduce the consumption of physical strength. In front of the net, No matter how many steps to the net, the teachers must emphasize the last step, it is that need to step foot toe abduction, heel touchdown, pull the bow and arrow step,focus on the front and rear legs, inertial momentum so as to offset the body forward, and effectively improve the return rate. Similarly, when back last step we should put the focus on the right leg, with the right pedal, at the same time, the left foot in the air to a cross action, put the right foot forward after swing, put the focus of front left foot pedal forward, so you can make a homeopathy return.

\section{Pay Attention to Cultivating the Step Rhythm of Students}

Without training, we will usually start slow in the badminton, resulting in step slow, and then lose the best chance. In the footwork teaching, pay particular attention to strengthening and cultivating the ability of the start. Improve starting speed, the key is to learn to make full use of ground movements of ankle joint strength, to obtain the first step power, it produces a kind of the response action that suddenly erupted from the relatively static state. in order to improve the ankle and leg strength,we can do rope skipping exercises and experience the pedaling force action. Do some manual starting footwork exercises, students can according to the teacher's instructions, follow the gestures direction to do front, midfielder and back court starting footwork exercise repeatedly, improve students' ability of rapid start. In the footwork of teaching, teachers should teach the students how to judge the direction, grasp the starting time, do well control of step reverse rhythm, neither too fast, nor too slow, the best starting time is when taken away from the ball support surface from the other side of the instant.students should be stressed in the timing and pace of starting at each practice time when the opponent batting, just complete judgment, that avoid starting moves too fast or too slow.

\section{Conclusion}

Badminton teaching should run through the guiding ideology of combination of methods and footwork, follow the principle that from simple to complex, from easy to difficult, step by step hitting footwork practice as the foundation and footwork combining practice as the focus by the targeted teaching, Teachers should pay attention to study and master the various footwork comprehensively, to strengthen and improve the students' ability to run the court by various means and to strengthen the various steps start and adjust skills training and improve the training. Hence the students hold present flexible use of footworks and improve the level of badminton technique and tactics constantly.

\section{References}

[1] Jie XIAO, Badminton Sports Theory and Practice[M], People's Sports Press

[2] Hasegawa Hiromiyuki, Badminton Hundred Answer[M],People's Sports Press

[3] Changhao JIANG, University Badminton Footwork Teaching in Specialized Course, Journal of Ningbo University[J], 2008

[4] Qiuming FENG, Moving Ability on Volleyball Sporting, Journal of Sports Adult Education [J], 2003-4

[5] Jingkang SHI, Introduction to Badminton Footwork Training and Its Importance, Sports Research and Education [J], 2013-1

[6] Suping WANG, Min PENG, The Focus and Principles of the Badminton Footwork Teaching , Liaoning Sport Science and Technology [J], 2002-4 